related hemorrhage in the FD group $(3.8 \%)$ and one in the coil group $(1.9 \%)$, both resulted in moderate morbidity (mRS 3 and 2, respectively), without mortality occurring.

Conclusion Flow diverters demonstrated a nearly twice higher rate of definitive angiographic cure vs coils, but the rate of clinically acceptable occlusion was similar. The complications and morbidity rates were equally very low. We suggest that the aneurysm and parent vessel anatomy should be the main factors when choosing the modality for treating SHA aneurysm.

Disclosures A. Gorbatykh: None. K. Orlov: None.

\section{E-184 NON-DESTRUCTIVE RHEOLOGY TECHNIQUE FOR MATERIAL CHARACTERIZATION OF CADAVERIC TISSUE}

N Nau*. Nau Dr. Becker, Flagstaff, AZ

10.1136/neurintsurg-2020-SNIS.215

Introduction/Purpose Although current vessel-training models cast from human vasculature are anatomically accurate, the materials used (i.e. silicones and glass) do not accurately simulate the vascular compliance (modulus) and wall friction effects (lubricity) seen in human vasculature. Deriving mechanical properties from samples historically results in destroying the samples being tested. Mechanical testing often delaminated tissue layers, ruptures the endotheliatl layer, or tears tissue completely. Mechanical characterization using dynamic loading (hybrid rheometer (DHR-2, TA Instruments) can provide reliable and repeatable material property data without damaging the tissue. Therefore, the same tissue samples can also undergo histological analysis of microstructures and results can be directly correlated to the mechanical characterization data. This ultimately reduces the samples needed to obtain statistically significant results.

Materials and Methods Non-destructive mechanical testing was performed on $8 \mathrm{~mm}$ diameter tissue samples (figure 1A). The following non-destructive tests were completed on the same samples: dynamic compressive modulus, shear modulus, tensile modulus, and poisson's ratio (figure 1B). Next, these samples were fixed in formalin, embedded, cross-sectioned, stained with $\mathrm{H} \& \mathrm{E}$, and scanned with a microscope scanner (resolutions from $1 \mathrm{x}$ to $40 \mathrm{x}$ magnification). The histological images were compared to control tissue samples taken from the same tissue (not mechanically tested, but histologically stained).
Properties of cadaveric vessels were compared and validated with histology. All layers of the vasculature were scanned for damage.

Results Gross inspection of the tissues showed no apparent damage, and the control and mechanically tested samples appeared identical. No apparent tissue damage was seen from the histologically prepped tissue slides (figure 1C and D). The endothelial layer remained intact on all tested and control samples. No delamination of tissue layers was observed and no histology artifacts were found on the tissue slides.

Conclusion Dynamic, non-destructive mechanical testing using rheological testing techniques is an effective approach to analyzing delicate and rare tissue samples without causing permanent damage. In the case of human vasculature, there is an opportunity to understand the anatomical and mechanical properties of diseased, normal, or calcified regions of tissue, even from the same sample. Reducing the need for repetitive testing on multiple human and animal tissue samples. Correlating mechanical and histological tissue properties directly can improve the creation of more accurate vessel analogs and invitro vessel models, ultimately improving device testing outcomes and reducing the need for biological samples.

Disclosures N. Nau: None.

\section{E-185 MR VESSEL WALL IMAGING IN THE DIAGNOSIS OF OCCULT RUPTURED BLISTER INTRACRANIAL ANEURYSMS}

${ }^{1} Y$ Moazeni ${ }^{*},{ }^{1} \mathrm{R}$ Abdalla, ${ }^{2} \mathrm{M}$ Sukumaran, ${ }^{1} \mathrm{D}$ Cantrell, ${ }^{3} \mathrm{~A}$ Shaibani, ${ }^{3} \mathrm{M}$ Hurley, ${ }^{3} \mathrm{~B}$ Jahromi, ${ }^{3} \mathrm{M}$ Potts, ${ }^{4} \mathrm{~S}$ Ansari. ${ }^{1}$ Department of Radiology, Northwestern Memorial Hospital, Feinberg School of Medicine of Northwestern University, Chicago, IL; ${ }^{2}$ Department of Neurological Surgery, Northwestern Memorial Hospital, Feinberg School of Medicine of Northwestern University, Chicago, IL; ${ }^{3}$ Departments of Radiology and Neurological Surgery, Northwestern Memorial Hospital, Feinberg School of Medicine of Northwestern University, Chicago, IL; ${ }^{4}$ Departments of Radiology, Neurological Surgery and Neurology, Northwestern Memorial Hospital, Feinberg School of Medicine of Northwestern University, Chicago, IL

\subsection{6/neurintsurg-2020-SNIS.216}

Purpose Blister intracranial aneurysms are a rare etiology of subarachnoid hemorrhage (SAH) accompanied by high morbidity and mortality rates. Digital subtraction angiography (DSA) and CT angiography (CTA) serve as the main imaging techniques for diagnosing the cause of $\mathrm{SAH}$, yet blister aneurysms can remain occult on DSA or CTA imaging due to their small size, broad based morphology, and early transient thrombosis.
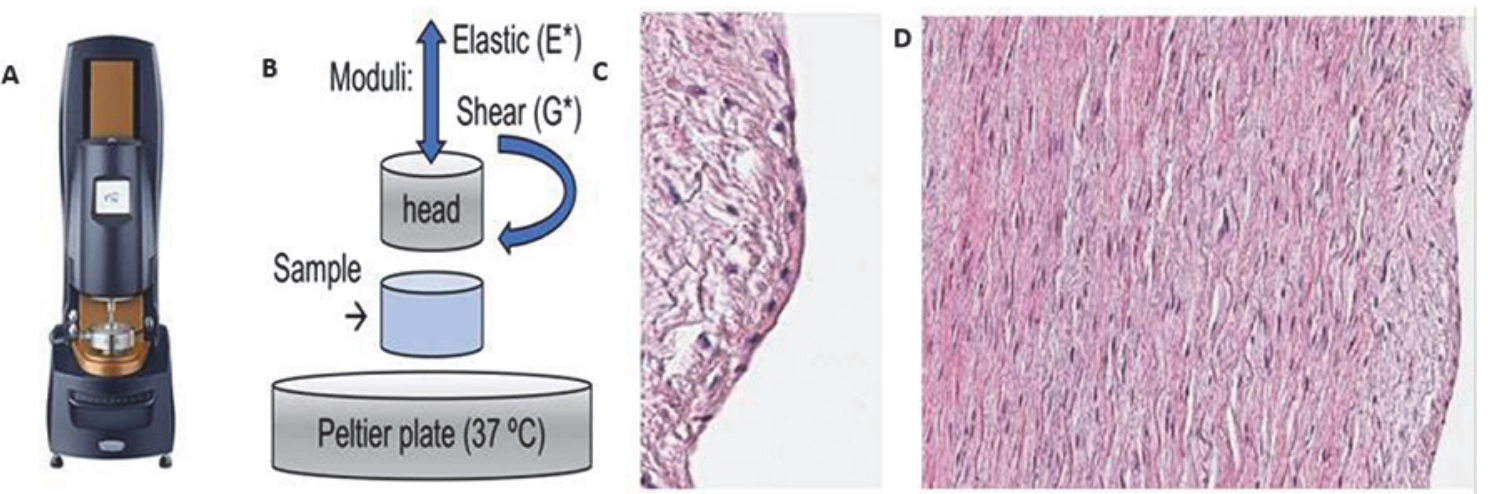

Abstract E-184 Figure 1 A-Hybrid rheometer: B-Rheometer setup for mechanical testing F C-undamaged vessel endothelial layer (40X) D-Histological slide of an undamaged human vessel 